\title{
The Formation of Singularities in the Harmonic Map Heat Flow
}

\author{
MATthew Grayson ${ }^{1}$ and Richard S. Hamilton ${ }^{2}$
}

Let $M$ and $N$ be compact Riemannian manifolds and let $F: M \rightarrow N$ be a map from one to the other. We can deform $F$ by its intrinsic Laplacian to smooth the map out; the resulting equation

$$
\frac{\partial F}{\partial t}=\Delta F
$$

is called the harmonic map heat flow. In their paper [3], Eells and Sampson introduced this flow and proved that if the target $N$ has non-positive sectional curvature, then the solution exists for all time; and a subsequence converges to a harmonic map $F$, one with $\Delta F=0$. When $N$ has curvature of unrestricted sign, singularities may develop, and it is interesting to see what form they may take. Partial answers have been given by Struwe in the case [10] where $\operatorname{dim} M=2$ and in the case [11] where $M=R^{m}$ is an Euclidean space. In the latter paper Struwe introduces an important monotonicity principle. In [4] we generalize Struwe's argument to the case of a curved domain. This involves some nontrivial estimates on the matrix of second derivatives of the heat kernel on a curved space, which are given in [5]. Using the monotonicity formula and local estimates on the higher derivatives $D^{k} F$ of the map derived in section 2 (following the line of WanXiong Shi in [8] and [9]) we rederive Struwe's $\varepsilon$-regularity result in section 3 and the following partial regularity result in section 4 (see [11] and [2]).

Theorem 0.1. Let $F$ be a solution of the harmonic map heat flow from $M^{m}$ to $N^{n}$ on $0 \leq t<T$ with energy bounded by $E_{0}$. There exists a closed set $S$ with finite $m-2$ dimensional Hausdorff measure such that $F(x, t)$ converges smoothly y to a limit $F(x, T)$ as $t \rightarrow T$ on compact sets in $M-S$. Moreover there exists a constant $C$ depending only on $M, N$ and $E_{0}$, such

\footnotetext{
${ }^{1}$ Partially supported by NSF contract DMS 89-05937

${ }^{2}$ Partially supported by NSF contract DMS 90-03333
} 
that if $U$ is any open set containing $S$ then

$$
\mathcal{H}_{m-2}(S) \leq C \lim _{t \rightarrow T} \inf \int_{U}|D F|^{2} .
$$

We then consider blow-ups of the solution at points $X$ in the singular set $S$. The point $X$ is singular if $F(x, t)$ does not converge smoothly to a limit in a neighborhood of $X$ as $t \rightarrow T$. If there are no singular points, the solution can be extended past $T$. Hence either the solution exists for all time, or there is some $x$ and some $T$ where the solution is singular. We prove the following result for rapidly forming singularities in section $S$.

Theorem Main Theorem. Let $F$ be a smooth solution of the Harmonic Map heat flow on $0 \leq t<T$ with a singularity at some point $X$ as $t \rightarrow T$. Suppose the singularity forms rapidly, so that

$$
(T-t)|D F|^{2} \leq C
$$

for some constant $C<\infty$. Then the sequence of blow-ups at $X$ by factor $\lambda$

$$
F \lambda(x, \tau)=F\left(\exp _{X} \lambda x, T+\lambda^{2} \tau\right)
$$

defined for tangent vectors $x$ at $X$ and rescaled time $\tau<0$ has a subsequence $\lambda_{i} \rightarrow 0$ for which the maps $F_{\lambda_{i}}$ converge in $\mathcal{C}^{\infty}$ on compact sets to a map $\bar{F}(x, \tau)$ defined on all of $T_{X} M \times(-\infty, 0)$ with values in $N$ solving the harmonic map heat flow equation also. The limit map $\bar{F}$ is dilation-invariant, so that

$$
\bar{F}\left(\lambda x, \lambda^{2} \tau\right)=\bar{F}(x, \tau) .
$$

We call such a map a homothetically shrinking solition. Moreover if $X$ is a singular point (i.e. if $F$ does not extend smoothly to a neighborhood of $X$ for $t \leq T$ ) then $\bar{F}$ is not constant; in particular there exists $\rho>0$ and $\varepsilon>0$ depending only on $M, N$, and the initial energy $E_{0}$ so that

$$
\int_{|x| \leq \rho}|D \bar{F}(x,-1)|^{2} e^{-|x|^{2} / 4} d x \geq \varepsilon .
$$

$\S 1$.We begin the paper with a computer study giving an example of a rapidly forming singularity for a map of $S^{3}$ to $S^{3}$, and show how it is asymptotic to a homothetically shrinking soliton mapping $R^{3}$ to a hemisphere of $S^{3}$. Moreover, the computer continues the solution past the singularity, and as the singularity smooths out it is asymptotic to a homothetically expanding soliton. 
We confine our attention to maps of $S^{n}$ to $S^{n}$ which are rotationally symmetric around a north and south pole. In the study we take $n=3$, but $n>3$ should look similar. We let $r$ denote the distance along the first sphere from the north pole, and $R$ the same distance on the second sphere. The Riemannian metrics on the spheres are

$$
d s^{2}=d r^{2}+\sin ^{2} r d \theta^{2} \text { and } d S^{2}=d R^{2}+\sin ^{2} R d \theta^{2}
$$

where $d \theta$ is the metric on the equatorial sphere $S^{n-1}$. Our map will have the form

$$
F(r, \theta, t)=(R(r, t), \theta)
$$

and the evolution can be expressed in terms of the function $R=R(r, t)$. We will have the north pole go to the north pole and the south pole go to the south pole, so $R=0$ at $r=0$ and $R=\tau$ at $r=\tau$, until the singularity forms. At the critical time $T$ we get $R=\tau / 2$ at $r=0$, and immediately after we jump to $R=\tau$ at $r=0$.

The energy map $F$ is

$$
E=\frac{1}{2} \operatorname{Vol}\left(S^{n-1}\right) \int\left[\left(\frac{\partial R}{\partial r}\right)^{2}+(n-1)\left(\frac{\sin R}{\sin r}\right)^{2}\right] d r
$$

and from this we can compute the harmonic map evolution of $R$, which is

$$
\frac{\partial R}{\partial t}=\Delta R-(n-1) \frac{\sin 2 R}{2 \sin r}
$$

where we use the spherical Laplacian

$$
\triangle R=\frac{\partial^{2} R}{\partial r^{2}}+(n-1) \frac{\cos r}{\sin r} \frac{\partial R}{\partial r} .
$$

We start the evolution with a function $R$ which is close to the identity map but shifted slightly toward the south pole. Since the identity is an unstable critical point of the harmonic map energy, the solution begins to drift south. As we approach the critical time $T$ a very small neighborhood of the north pole in the domain maps to cover the entire northern hemisphere in the target. At the critical time $T$ the map $F$ is discontinuous at the north pole, and its image covers only the southern hemisphere. Subsequently the map closes up, so that it covers almost all of the southern hemisphere twice. As time evolves, the region covered twice shrinks toward the south pole, and no further pathology happens. 
The physical reason for the flip is that it takes very little energy to map a small ball to a hemisphere in dimension $n \geq 3$. The solitons can best be seen by graphing $R$ as a function of $\frac{1}{r}$ and $t$; as $t$ increases to $T$ the function $R$ for $r \simeq 0$, corresponding to $\log \frac{1}{r} \simeq+\infty$, looks like a wave traveling forward, while for $t$ past $T$ the function $R$ looks like a wave traveling backwards. In the rest of this paper we only consider the formation of singularities; but the computer study suggests there are similar models for their dissipation. Here we exhibit the graphs coming from the computer.

Very different behavior happens when the domain $M$ has dimension 2. In this case there are no homothetically shrinking solitions, and all singularities form slowly, i.e. $(T-t)|D F|^{2}$ is unbounded. Nevertheless they do form in finite time, and they are asymptotic to a harmonic map of $R^{2}$ into the target. (See Struwe [10] for a proof that the singularities are isolated points in space-time, and the recent work of Chang, Ding, and Ye [1] for an example of a singularity on a surface forming in finite time.) We can get similar phenomena in higher dimensions by crossing with a flat $R^{k}$ in the domain, with no change in the map. Hence slowly forming singularities can form in any dimension $m \geq 2$. We would analyze them also. It seems reasonable to conjecture that for any slowly forming singularity there is a sequence of dilations of the solution which converges either to a harmonic map, or to a translating solition, i.e. a solution of the harmonic map heat flow

$$
\vec{F}: T_{X} M \times(-\infty, \infty) \rightarrow N
$$

such that there is some vector $v$ such that

$$
\bar{F}(x, t)=\bar{F}(x+t v, 0) .
$$

Of course in case $v=0$ the soliton is just a stationary harmonic map. It would be interesting to exhibit such a translating soliton with $v \neq 0$, and very interesting to show hoe it arises as a limit of dilations of solutions coming from a compact domain $M$.

$\S 2$.Next we prove local bound on the higher derivatives of a solution to the harmonic map heat flow. In the sequel we will use parabolic cylinders

$$
P_{r}(X, T)=\left\{(x, t) \in M \times R: d(X, x) \leq r \text { and } T-r^{2} \leq t \leq T\right\}
$$

We start with a useful lemma on scalar functions.

Lemma 2.1. Let $M$ be a compact be a compact manifold. There exists a constant $s>0$, and for every $\gamma<1$ a constant $C_{\gamma}$ such that if $h$ is a smooth function satisfying

$$
\frac{\partial h}{\partial t} \leq \triangle h-h^{2}
$$


when $h \geq 0$ in some parabolic cylinder $P_{r}(X, T)$ around some $X \in M$ with $r \leq s$ then

$$
h \leq C_{\gamma}\left(\frac{1}{r^{2}}+\frac{1}{t}\right)
$$

on $P_{\gamma r}(X, T)$.

Proof. First we show how to construct a function $\varphi$ with support in the closed ball $\bar{B}_{r}(X)$ of radius $r$ around $X$ such that we have the estimates

$$
\begin{gathered}
\varphi(X) \geq c r \\
0 \leq \varphi \leq C r \\
|D \varphi| \leq C_{1} \\
\left|D^{2} \varphi\right| \leq C_{2} / r
\end{gathered}
$$

for some constants $c>0$ and $C, C_{1}, C_{2}<\infty$ independent of $r$ and $X$, depending only on $M$. For any point in $M$ we can find a chart $\lambda$ on $M$ defined on the open ball $B_{3}$ of radius 3 in $R^{m}$ mapping 0 to the point. We can find a finite number of such charts $\left\{\lambda_{\alpha}\right\}$ such that the images of the open ball $B_{1}$ of radius 1 cover all of $M$. We can find constants $K_{1}, K_{2}$ and $K$ such that for all $\alpha$

$$
\left|D \lambda_{\alpha}\right| \leq K_{1},\left|D^{2} \lambda_{\alpha}\right| \leq K_{2} \text {, and }\left|D \lambda_{\alpha}^{-1}\right| \leq K
$$

on the closed ball $\bar{B}_{2}$ of radius 2 .

Given any $X \in M$ we can find an $\alpha$ and a $V \in B_{1}$ with $\lambda_{\alpha}(V)=X$. Take $s \leq K_{1}$ and $r \leq s$, and let $\rho=r / K_{1}$. If $\bar{B}_{\rho}(V)$ is the closed ball of radius $\rho$ around $V$ then $\bar{B}_{\rho}(V) \subseteq \bar{B}_{2}$ since $\rho \leq 1$. Also $\lambda_{\alpha}$ maps $\bar{B}_{\rho}(V)$ into $\bar{B}_{r}(X)$ since $\left|D \lambda_{\alpha}\right| \leq K_{1}$. Choose any function $\omega$ with support in $B_{1}$ with $\omega \geq 0$ and $\omega(0)>0$. Then we have

$$
\begin{gathered}
\omega(0) \geq c \\
0 \leq \omega \leq C \\
|D \omega| \leq C_{1} \\
\left|D^{2} \omega\right| \leq C_{2}
\end{gathered}
$$

for some constants $c>0$ and $C, C_{1}, C_{2}<\infty$. We translate and rescale $\omega$ to $\bar{B}_{\rho}(V)$ by letting

$$
\omega^{*}(v)=\rho \omega([v-V] / \rho) .
$$


Then we have

$$
\begin{gathered}
\omega^{*}(V) \geq c \rho \\
0 \leq \omega^{*} \leq C \rho \\
0 \leq\left|D \omega^{*}\right| \leq C_{1} \\
0 \leq\left|D^{2} \omega^{*}\right| \leq C_{2} / \rho .
\end{gathered}
$$

Now define $\varphi$ by the pull back

$$
\varphi=\omega^{*} \circ \lambda_{\alpha}^{-1} .
$$

Then $\varphi$ has support in $\bar{B}_{r}(X)$ and we can check that

$$
\begin{gathered}
\varphi(X) \geq c r / K_{1} \\
0 \leq \varphi \leq C r / K_{1} \\
0 \leq|D \varphi| \leq C_{1} K \\
0 \leq\left|D^{2} \varphi\right| \leq C_{2} K_{1} K_{2} K^{2} / r
\end{gathered}
$$

which proves our claim with slightly different constants.

In particular we can find a constant $A$ such that

$$
6|D \varphi|^{2}-2 \varphi \triangle \varphi \leq A \text {. }
$$

Then we let

$$
\psi=\frac{A}{\varphi^{2}}+\frac{1}{t}
$$

and compute

$$
\triangle \psi=A\left[6|D \varphi|^{2}-2 \varphi \Delta \varphi\right] / \varphi^{4} \leq A^{2} / \varphi^{4} .
$$

Since

$$
\left(\frac{A}{\varphi^{2}}+\frac{1}{t}\right)^{2} \geq \frac{A^{2}}{\varphi^{4}}+\frac{1}{t^{2}}
$$

we have

$$
\frac{\partial \psi}{\partial t} \geq \Delta \psi-\psi^{2} .
$$

But

$$
\frac{\partial h}{\partial t} \leq \Delta h-h^{2}
$$

when $h \geq 0$ so if $k=\psi-h$ we have for $k \geq 0$

$$
\frac{\partial k}{\partial t} \geq \Delta k-(h+\psi) k \text {. }
$$


Now $k$ is a smooth function in the interior of the support of $\varphi$ for $t>0$, and $k \rightarrow+\infty$ as $t \rightarrow 0$ or as we approach the boundary of the support of $\varphi$. Therefore the maximum principle shows $k \geq 0$ for all $t>0$. This means $h \leq \psi$. Since we chose $\varphi$ with $\varphi(X) \geq c r$, we have

$$
h(X, t) \leq \psi(X, t) \leq C\left(\frac{1}{r^{2}}+\frac{1}{t}\right) .
$$

We now obtain the desired result by replacing $X$ by any point in $\bar{B}_{\gamma r}(X)$ for $\gamma<1$ and adjusting the constant. Or equivalently we can make $\varphi \geq c r$ in $\bar{B}_{\gamma r}(X)$ by making $\omega \geq c$ in $\bar{B}_{\gamma}$.

We apply the lemma to derive local bounds on the higher derivatives of a solution of the harmonic map heat flow.

Theorem 2.2. There exists a constant $s>0$ depending only on $M$, and constants $C_{k}$ for $k=2,3, \cdots$ depending only on $M$ and $N$, such that if $F$ is any solution to the harmonic map heat flow with $|D F| \leq A$ in some parabolic cylinder $P_{r}(X, T)$ for $r \leq s$ for some constant $A \geq 1 / r$, then with $r_{k}=r / 2^{k-1}$ we have

$$
\left|D^{k} F\right| \leq C_{k} A^{k} \text { onP } P_{r_{k}}(X, T) .
$$

Proof. We start with the case $k=2$. We have formulas of the form

$$
\frac{\partial}{\partial t} D F=\triangle D F+R_{M} * D F+R_{N}(F) * D F^{3}
$$

where $R_{M}$ and $R_{N}$ are the curvature tensors on $M$ and $N$, and where * denotes some linear tensor contraction, possible including constants. Differentiating

$$
\begin{aligned}
\frac{\partial}{\partial t} D^{2} F=\triangle D^{2} F & +R_{M} * D^{2} F+R_{N}(F) * D F^{2} * D^{2} F \\
& +D R_{M} * D F+D R_{N}(F) * D F^{4}
\end{aligned}
$$

Using $|D F| \leq A$ we get estimates

$$
\begin{aligned}
\frac{\partial}{\partial t}|D F|^{2} & \leq \triangle|D F|^{2}-2\left|D^{2} F\right|^{2}+C A^{4} \\
\frac{\partial}{\partial t}\left|D^{2} F\right|^{2} & \leq \triangle\left|D^{2} F\right|^{2}-2\left|D^{3} F\right|^{2}+C A^{2}\left|D^{2} F\right|^{2}+C A^{6}
\end{aligned}
$$


where in the latter case we use

$$
A^{4}\left|D^{2} F\right| \leq A^{2}\left|D^{2} F\right|^{2}+A^{6} .
$$

Now we let

$$
h=\left(8 A^{2}+|D F|^{2}\right)\left|D^{2} F\right|^{2}
$$

and compute

$$
\begin{aligned}
\frac{\partial h}{\partial t} \leq & \left(8 A^{2}+|D F|^{2}\right) \triangle\left|D^{2} F\right|^{2}+\left|D^{2} F\right|^{2} \triangle|D F|^{2} \\
& -16 A^{2}\left|D^{3} F\right|^{2}-2\left|D^{2} F\right|^{4}+C A^{4}\left|D^{2} F\right|^{2}+C A^{8} .
\end{aligned}
$$

Also we have

$$
\triangle h \geq\left(8 A^{2}+|D F|^{2}\right) \triangle\left|D^{2} F\right|^{2}+\left|D^{2} F\right|^{2} \triangle|D F|^{2}-8 A\left|D^{2} F\right|^{2}\left|D^{3} F\right|
$$

and

$$
8 A\left|D^{2} F\right|^{2}\left|D^{3} F\right| \leq 16 A^{2}\left|D^{3} F\right|^{2}+\left|D^{2} F\right|^{4}
$$

so we get

$$
\frac{\partial h}{\partial t} \leq \triangle h-\left|D^{2} F\right|^{4}+C A^{4}\left|D^{2} F\right|^{2}+C A^{8} .
$$

Since $h \leq 8 A^{2}\left|D^{2} F\right|^{2}$ we have

$$
\frac{\partial h}{\partial t} \leq \Delta h-\frac{h^{2}}{C A^{4}}+C A^{8}
$$

for some constant $C$. Then let

$$
h=C A^{4} \tilde{h}+C A^{6}
$$

for the same constant $C$, and we get for $\tilde{h} \geq 0$

$$
\frac{\partial \tilde{h}}{\partial t} \leq \Delta \tilde{h}-\tilde{h}^{2} .
$$

We can now apply Lemma 2.1 to conclude

$$
\tilde{h} \leq C / r^{2}
$$

on $P_{r / 2}(X, T)$. This makes

$$
h \leq C A^{6}
$$

since $A \geq 1 / r$, and we conclude that

$$
\left|D^{2} F\right| \leq C A^{2}
$$


on $P_{r / 2}(X, T)$.

To bound the third derivative we follow the same procedure. Now we have a formula

$$
\begin{aligned}
\frac{\partial}{\partial t} D^{3} F & =\triangle D^{3} F+R+M * D^{3} F+R_{N}(F) * D F^{2} * D s F \\
& +D R_{M} * D^{2} F+D R_{N}(F) * D F^{3} * D^{2} F \\
& +R_{N}(F) * D F * D^{2} F^{2}+D^{2} R_{M} * D F \\
& +D^{2} R_{N}(F) * D F^{5}
\end{aligned}
$$

and hence with $\left|D^{2} F\right| \leq C A^{2}$ on $P_{r / 2}(X, T)$ we have

$$
\frac{\partial}{\partial t}\left|D^{3} F\right|^{2} \leq \triangle\left|D^{3} F\right|^{2}-2\left|D^{4} F\right|^{2}+C A^{2}\left|D^{3} F\right|^{2}+C A^{8}
$$

and also

$$
\frac{\partial}{\partial t}\left|D^{2} F\right|^{2} \leq \triangle\left|D^{2} F\right|^{2}-2\left|D^{3} F\right|^{2}+C A^{6}
$$

from our previous bound and the new bound on $\left|D^{2} F\right|^{2}$. Then letting $B$ be a constant with $A^{2} \leq B \leq C A^{2}$ and $\left|D^{2} F\right| \leq B$ and

$$
h=\left(8 B^{2}+\left|D^{2} F\right|^{2}\right)\left|D^{3} F\right|^{2} .
$$

We have

$$
\begin{aligned}
\frac{\partial h}{\partial t} \leq & \left(8 B^{2}+\left|D^{2} F\right|^{2}\right) \triangle\left|D^{3} F\right|^{2}+\left|D^{3} F\right|^{2} \triangle\left|D^{2} F\right|^{2} \\
& -16 B^{2}\left|D^{4} F\right|^{2}-2\left|D^{3} F\right|^{4}+C A^{6}\left|D^{3} F\right|^{2}+C A^{12} .
\end{aligned}
$$

Also

$$
\triangle h \geq\left(8 B^{2}+\left|D^{2} F\right|^{2}\right) \triangle\left|D^{3} F\right|^{2}+\left|D^{3} F\right|^{2} \triangle\left|D^{2} F\right|^{2}-8 B\left|D^{3} F\right|^{2}\left|D^{4} F\right|
$$

and using

$$
8 B\left|D^{3} F\right|^{2}\left|D^{4} F\right| \leq 16 B^{2}\left|D^{4} F\right|^{2}+\left|D^{3} F\right|^{4}
$$

we conclude

$$
\frac{\partial h}{\partial t} \leq \triangle h-\left|D^{3} F\right|^{4}+C A^{6}\left|D^{3} F\right|^{2}+C A^{12} .
$$

Since $h \geq 8 B^{2}\left|D^{3} F\right|^{2}$ and $B \geq A^{2}$ we have

$$
\frac{\partial h}{\partial t} \leq \triangle h-\frac{h^{2}}{C A^{8}}+C A^{12}
$$


for some constant $C$. Then let

$$
h=C A^{8} \tilde{h}+C A^{10}
$$

and we get

$$
\frac{\partial \tilde{h}}{\partial t} \leq \triangle \tilde{h}-\tilde{h}^{2}
$$

and conclude that on $P_{r / 4}(X, T)$ we have $\tilde{h} \leq C / r^{2} \leq C A^{2}$. This makes

$$
\left|D^{3} F\right| \leq C A^{3}
$$

on $P_{r / 4}(X, T)$. The higher derivatives can be handled in the same way, by induction.

§3.Now we prove an $\varepsilon$-regularity result similar to that in Struwe [11]. We start again with a lemma. For any $X$ and $T$ and any $r>0$ we define as before the parabolic cylinder of radius $R$ around $(X, T)$ by

$$
P_{r}(X, T)=\left\{(x, t): d(x, X) \leq r, T-r^{2} \leq t \leq T\right\} .
$$

and we let $k(\xi, \tau)$ be the backwards solution of the heat equation on $M$ which becomes a delta function at the point $\xi$ at time $\tau$.

Lemma 3.1. There exist constants $\delta>0$ and $\gamma$ in $0<\gamma<1$ depending only on the manifolds $M$ and $N$, with the property that if $F$ is any solution of the harmonic map heat flow in a parabolic cylinder $P_{r}(\xi, \tau)$ of radius $r \leq 1$ around a point $\xi \in M$ and time $\tau \in R$ with values in $N$ such that

$$
|D F(\xi, \tau)|=1 / r
$$

and

$$
|D F(x, t)| \leq 2 / r
$$

for all $(x, t) \in P_{r}(\xi, \tau)$ then for $\theta=\tau-\gamma r^{2}$ we have

$$
(\tau-\theta) \int_{M}|D F(x, \theta)|^{2} k_{(\xi, \tau)}(x, \theta) d x \geq \delta .
$$

Proof. By the local estimate on higher derivatives given by Theorem 2.2 we can find constants depending only on $M$ and $N$ such that

$$
\mid D^{2} F(x, t) \leq C / r^{2} \text { and }\left|D^{3} D(x, t)\right| \leq C / r^{3}
$$


for all $(x, t) \in P_{r / 2}(\xi, \tau)$. Then since

$$
\frac{\partial}{\partial t} D F=\triangle D F+R_{M} * D F+R_{N} * D F^{3}
$$

we have

$$
\left|\frac{\partial}{\partial t} D F(x, t)\right| \leq C / r^{3}
$$

for all $(x, t) \in P_{r / 2}(\xi, \tau)$ also. These bounds on the space and time derivatives of $D F$ show that

$$
|D F(x, t)| \geq 1 / 2 r
$$

for all $(x, t) \in P_{\gamma r}(\xi, \tau)$ for some $\gamma>0$. On the other hand, by Corollary 2.3 of [H2] we have

$$
k_{(\xi, \tau)}(x, \theta) \geq c / r^{m}
$$

for some constant $c>0$ depending only on $M$ for all $x$ in the ball of radius $\gamma r$ around $\xi$, with $\theta=\tau-\gamma r^{2}$. Then

$$
(\tau-\theta) \int_{M}|D F(x, \theta)|^{2} k_{(\xi, \tau)}(x, \theta) d x \geq \delta
$$

for some $\delta>0$ as claimed, as we see from integrating just over the ball.

Theorem 3.2. Given $M, N$ and $E_{0}$, there exist constants $\varepsilon>0$ and $\beta>0$ such that for any $X \in M$, any $T>0$ and any $\alpha \geq 0$ in $T-\beta \leq \alpha<T$ we can find $\rho>0$ and $B<\infty$ so that if $F$ is any solution to the harmonic map heat flow from $M$ to $N$ on $0 \leq t \leq T$ with energy bounded by $E_{0}$ and

$$
(T-\alpha) \int_{M}|D F(x, \alpha)|^{2} k_{(X, T)}(x, \alpha) d x \leq \varepsilon
$$

then $|D F(x, t)| \leq B$ for all points $(x, t) \in P_{\rho}(X, T)$.

Proof. By Theorem 3.1 of [5], for any $\eta>0$ and any constant $C>1$ and any $X \in M$ and any $\tilde{T}$ with $0<\tilde{T} \leq 1$ we can find a $\rho>0$ such that any positive solution $f$ of the scalar forward heat equation with $\int f=1$ satisfies

$$
\tilde{\tau} f(\xi, \tilde{\tau}) \leq C \tilde{T} f(X, \tilde{T})+\eta / 2 E_{0}
$$

for all $(\xi, \tilde{\tau}) \in P_{\rho}(X, \tilde{\tau})$. For any $x$ let $f_{x}$ be the fundamental solution approaching a $\delta$-function at $x$ as $\tilde{t} \rightarrow 0$. Then with $\tilde{\tau}=\tau-\alpha$ and $\tilde{T}=T-\alpha$ we have

$$
k_{(\xi, \tau)}(x, \alpha)=f_{x}(\xi, \tilde{\tau}) \text { and } k_{(X, T)}(x, \alpha)=f_{x}(X, \tilde{T})
$$


by the symmetry of the heat kernel. Therefore

$$
(\tau-\alpha) k_{(\xi, \tau)}(x, t) \leq C(T-\alpha) k_{(X, T)}(x, t)+\eta / 2 E_{0}
$$

and we conclude that

$$
(\tau-\alpha) \int_{M}|D F(x, \alpha)|^{2} k_{(\xi, \tau)}(x, \alpha) d x \leq \eta
$$

for all $(\xi, \tau) \in P_{\rho}(X, T)$ provided that we choose $\varepsilon>0$ with $C \varepsilon \leq \eta / 2$.

Using this $\rho$ we define

$$
\varphi(x, t)=\min \left\{\rho-d(X, x), \sqrt{\rho^{2}-(T-t)}\right\}
$$

and consider the function

$$
\varphi(x, t)|D F(x, t)|
$$

on $P_{\rho}(X, T)$. It will attain its maximum at some point $(\xi, \tau)$ with $\varphi(\xi, \tau)>$ 0 . Let $\sigma=\varphi(\xi, \tau)$ and let

$$
A=\sigma|D F(\xi, \tau)|
$$

It is easy to check that

$$
P_{\sigma}(\xi, \tau) \subseteq P_{\rho}(X, T)
$$

and that

$$
\varphi(x, t) \geq \sigma / 2 \text { for }(x, t) \in P_{\sigma / 2}(\xi, \tau) .
$$

Consequently if $r=\sigma / A$

$$
|D F(\xi, \tau)| \leq 2 A / \sigma=1 / r
$$

and

$$
|D F(x, t)| \leq 2 A / \sigma=2 / r
$$

when $(x, t) \in P_{r}(\xi, \tau)$, as long as $r \leq \sigma / 2$. Now if $r \geq \sigma / 2$, then $A=\sigma / r \leq$ 2 , and since $\varphi(x, t) \geq \rho / 2$ for $(x, t) \in P_{\rho / 2}(X, T)$ we get a bound $|D F(x, t)| \leq$ $B$ with $B=2 A / \rho$, finishing the theorem. Otherwise we continue by applying Lemma 3.1 to conclude that for some $\delta>0$ and $\gamma$ with $0<\gamma<1$ we have

$$
(\tau-\theta) \int_{M}|D F(x, \theta)|^{2} k_{(\xi, \tau)}(x, \theta) d x \geq \delta
$$


with $\theta=\tau-\gamma r$. Since $r \leq \sigma / 2, \theta \geq \tau-\sigma / 2$. Now the expression

$$
Z(t)=(\tau-t) \int_{M}|D F(x, t)|^{2} k_{(\xi, \tau)}(x, t) d x
$$

satisfies the monotonicity principle of Corollary 1.4; we can find a constant $C$ such that

$$
Z(\theta) \leq C Z(\alpha)+C E_{0}(\theta-\alpha) .
$$

We know that $\theta-\alpha \leq T-\alpha \leq \beta$ and $\beta$ can be chosen so small that $C E_{0} \beta<\delta / 2$. We can also choose $\eta$ so small that $c \eta<\delta / 2$. Then since $Z(\alpha) \leq \eta$ we get $Z(\theta)<\delta$, which is a contradiction. This completes the proof.

Corollary 3.3. Given $M, N$ and $E_{0}$, there exist and $\varepsilon>0$ and $a \beta>0$ such that if $F$ is any solution to the harmonic map heat flow from $M$ to $N$ on $0 \leq t<T$ and if

$$
(T-t) \int_{M}|D F(x, t)|^{2} k_{(X, T)}(x, t) d x \leq \varepsilon
$$

for some $t$ in $T-\beta \leq t<T$ then $F(x, t)$ extends smoothly on $0 \leq t \leq T$ in some neighborhood of $X$.

Proof. We apply the above estimate to the translates $\tilde{F}(x, t)=F(x, t-\zeta)$ for $\zeta>0$ and let $\zeta \rightarrow 0$. This gives uniform estimates on $|D F(x, t)|$ in some parabolic cylinder $P_{r}(X, T)$ uniform for $t<T$. The estimates on the higher derivatives then follow from the bound in Theorem 2.2.

$\S 4$. We recall the definition of $k$-dimensional Hausdorff measure. Let $M$ be a compact Riemannian manifold of dimension $m$. For any $k$ with $0 \leq k \leq m$ let $\mu_{k}$ be the volume of the unit ball in $R^{k}$ and define the $k$-dimensional measure of a set $S \subseteq M$ by

$$
\mathcal{H}_{k}(S)=\lim _{r \rightarrow 0} \inf _{x_{j}, r_{j}}\left\{\mu k \sum_{j} r_{j}^{k}: S \subseteq \bigcup B_{r_{j}}\left(x_{j}\right) \text { and } r_{j} \leq r\right\}
$$

where $B_{r}(x)$ is the geodesic ball of radius $r$ around $x$. We shall need the following lemma, which says that any set $S$ can be approximated by a good subset $\tilde{S}$. 
Lemma 4.1. For any $\eta>0$ and any set $S$ with $0<\mathcal{H}_{k}(S)<\infty$, we can find a subset $\tilde{S} \subseteq S$ such that

(1) $\mathcal{H}_{k}(\tilde{S}) \geq(1-2 \eta) \mathcal{H}_{k}(S)$, and

(2) $\exists r_{0}>0$ such that for any $r \leq r_{0}$ and any $x \in M$

$$
\mathcal{H}_{k}\left(B_{r}(x) \cap \tilde{S}\right) \leq\left(1+\frac{1}{\eta}\right) \mu_{k} r^{k}
$$

Proof. Pick $r_{0}$ so small that if $S \subseteq \bigcup_{j} B_{r_{j}}\left(x_{j}\right)$ with $r_{j} \leq r_{0}$ then

$$
\mu_{k} \sum_{j} r_{j}^{k} \geq(1-\eta) \mathcal{H}_{k}(S)
$$

as we can do from the definition. Now if possible we pick $r_{1}=r_{0}$ and pick $x_{1} \in M$ so that

$$
\mathcal{H}_{k}\left(B_{r_{1}}\left(x_{1}\right) \cap S\right) \geq \mu_{k} r_{1}^{k} / \eta
$$

If not, we take $r_{1}=r_{0} /(1+\eta)^{1 / k}$ and try again. We continue to diminish $r_{1}$ until we succeed;but if nor -1 works we stop the process. Otherwise we pick $r_{2}=r_{1}$ and try to pick $x_{2} \in M$ so that

$$
\mathcal{H}_{k}\left(B_{r_{2}}\left(x_{2}\right) \cap\left[S-B_{r_{1}}\left(x_{1}\right)\right]\right) \geq \mu_{k} r_{2}^{k} / \eta \text {. }
$$

If we cannot, we try $r_{2}=r_{1} /(1+\eta)^{1 / k}$, and continue to diminish $r_{2}$ until we succeed, but if no $r_{2}$ works we terminate the process. Continuing inductively, having chosen $r_{1}, \cdots, r_{j-1}$ and $x_{1}, \cdots, x_{j-1}$ we try $r_{j}=r_{j-1}$ or $r_{j}=r_{j-1} /(1+\eta)^{1 / k}$ or whatever so that

$$
\mathcal{H}_{k}\left(B_{r_{j}}\left(x_{j}\right) \cap\left[S-B_{r_{1}}\left(x_{1}\right)-\cdots-B_{r_{j-1}}\left(x_{j-1}\right)\right]\right) \geq \mu_{k} r_{j}^{k} / \eta
$$

and we continue the process as long as we can. Either it stops, or $r_{j} \rightarrow 0$ as $j \rightarrow \infty$ since $\mathcal{H}_{k}(S)<\infty$. Now we let

$$
\sum=S \cap\left[\bigcup_{j} B_{r_{j}}\left(x_{j}\right)\right] \text { and } \tilde{S}=S-\sum .
$$

The sets

$$
B_{r_{j}}\left(x_{j}\right) \cap\left[S-B_{r_{1}}\left(x_{1}\right)-\cdots-B_{r_{j-1}}\left(x_{j-1}\right)\right]
$$

form a finite or countable disjoint exhaustion of $\sum$, so

$$
\mathcal{H}_{k}\left(\sum\right) \geq \mu_{k}\left(\sum_{j} r_{j}^{k}\right) / \eta
$$


Moreover we can find balls $B_{s_{\ell}}\left(y_{1}\right)$ with $s_{\ell} \leq r_{0}$ which cover $\tilde{S}$ so that

$$
\mu_{k} \sum_{\ell} s_{\ell}^{k} \leq(1+\eta) \mathcal{H}_{k}(\tilde{S})
$$

by the definition. Since the balls $B_{r_{j}}\left(x_{j}\right)$ cover $\sum$, both sets of balls together cover $S=\tilde{S} \cup \sum$, so

$$
\mu_{k}\left[\sum_{j} r_{j}^{k}+\sum_{\ell} s_{\ell}^{k}\right] \geq(1-\eta) \mathcal{H}_{k}(S) .
$$

Combining these estimates gives

$$
(1-\eta) \mathcal{H}_{k}(S) \leq \eta \mathcal{H}_{k}\left(\sum\right)+(1+\eta) \mathcal{H}_{k}(\tilde{S})
$$

Now since $\mathcal{H}_{k}\left(\sum\right)=\mathcal{H}_{k}(S)-\mathcal{H}_{k}(\tilde{S})$ we get

$$
\mathcal{H}_{k}(\tilde{S}) \geq(1-2 \eta) \mathcal{H}_{k}(S)
$$

Finally it follows from the choice of the $r_{j}$ and $x_{j}$ that for any $r \leq r_{0}$ and any $x \in M$ we have

$$
\mathcal{H}_{k}\left(B_{r}(x) \cap \tilde{S}\right) \leq\left(1+\frac{1}{\eta}\right) \mu_{k} r^{k}
$$

The reason is that otherwise we would have chosen this ball instead. For if

$$
\mathcal{H}_{k}\left(B_{r}(x) \cap \tilde{S}\right)>\left(1+\frac{1}{\eta}\right) \mu_{k} r^{k}
$$

then when $r_{j} \leq r \leq r_{j} /(1+\eta)^{1 / k}$ we have

$$
\mathcal{H}_{k}\left(B_{r_{j}}(x) \cap \tilde{S}\right) \geq \mathcal{H}_{k}\left(B_{r}(x) \cap \tilde{S}\right) \geq\left(1+\frac{1}{\eta}\right) \mu_{k} r^{k} \geq \mu_{k} r_{j}^{k} / \eta
$$

and therefore this ball would have been chosen before reducing $r_{j}$.

Recall that $k_{y}(x, t)$ is the solution to the heat equation from a $\delta$-function at $y$ when $t=0$. For any set $S$ of finite $k$-dimensional measure we let

$$
k_{S}(x, t)=\int_{y \in S} k_{y}(x, t) d \mathcal{H}_{k}(y)
$$

be the solution to the heat equation emanating from the source $S$. 
Theorem 4.2. There exists a constant $C$ such that for any set $S$ of finite $k$-dimensional measure we can find a subset $\tilde{S}$ of $S$ with $\mathcal{H}_{k}(\tilde{S}) \geq \frac{1}{2} \mathcal{H}_{k}(S)$ and $a t_{0}>0$ such that for all $t \leq t_{0}$ and all $x \in M$

$$
k_{\tilde{S}}(x, t) \leq C / t^{(m-k) / 2} .
$$

Proof. We take $\eta=1 / 4$ in the previous lemma. Then for any $S$ we can find an $\tilde{S}$ with $\mathcal{H}_{k}(\tilde{S}) \geq \frac{1}{2} \mathcal{H}_{k}(S)$ and an $r_{0}>0$ such that for any $x$ and any $r \leq r_{0}$

$$
\mathcal{H}_{k}\left(B_{r}(x) \cap \tilde{S}\right) \leq 5 \mu_{k} r^{k}
$$

Now we need an upper bound on the heat kernel, such as is given in Corollary 3.1 of the paper by Li and Yau [7]; this shows that for any $\alpha<1$ there is a constant $C$ such that for $0<t \leq 1$

$$
k_{y}(x, t) \leq \frac{C}{t^{m / 2}} e^{-\alpha d(x, y)^{2} / 4 t} .
$$

Since $t \log (1 / t) \rightarrow 0$ as $t \rightarrow 0$, we can find $t_{0}>0$ such that if $t \leq t_{0}$ then

$$
t \log \mathcal{H}_{k}(\tilde{S})+\frac{k}{2} t \log (1 / t) \leq \alpha r_{0}^{2} / 4
$$

which is equivalent to

$$
\mathcal{H}_{k}(\tilde{S}) \cdot \frac{1}{t^{m / 2}} e^{-\alpha r_{0}^{2} / 4 t} \leq 1 / t^{(m-k) / 2} .
$$

This shows that for $t \leq t_{0}$

$$
\int_{y \in \tilde{S}-B_{r_{0}}(x)} k_{y}(x, t) d \mathcal{H}_{k}(y) \leq C / t^{(m-k) / 2}
$$

with a constant $C$ independent of $\tilde{S}$.

To estimate the rest of the integral, let $r_{j}=r_{0} / 2^{j}$ for $j \geq 0$ and let

$$
\tilde{S}_{j}=\left\{y \in \tilde{S}: r_{j} \leq d(x, y) \leq r_{j-1}\right\} .
$$

Then for $y \in \tilde{S}_{j}$ we have

$$
k_{y}(x, t) \leq \frac{C}{t^{m / 2}} e^{-\alpha r_{j}^{2} / 4 t}
$$


and since

$$
\mathcal{H}_{k}\left(\tilde{S}_{j}\right) \leq \mathcal{H}_{k}\left(B_{r_{j-1}}(x) \cap \tilde{S}\right) \leq 5 \mu_{k} r_{j-1}^{k}
$$

we get

$$
\int_{y \in \tilde{S}_{j}} k_{y}(x, t) d \mathcal{H}_{k}(y) \leq \frac{C}{t^{m / 2}} \cdot r_{j-1}^{k} e^{-\alpha r_{j}^{2} / 4 t} .
$$

If we use $r_{j-1}=2 r_{j}$ and let $u_{j}=r_{j} / \sqrt{t}$ then we get

$$
\sum_{j} \int_{y \in \tilde{S}_{j}} k_{y}(x, t) d \mathcal{H}_{k}(y) \leq \frac{C}{t^{(m-k) / 2}} \sum_{j=0}^{\infty} u_{j}^{k} e^{-\alpha u_{j}^{2} / 4}
$$

where $u_{j+1}=u_{j} / 2$. Now the latter sum is bounded independently of $u_{0}<$ $\infty$. This will prove the result.

To see the sum is bounded, we divide it into two parts. Suppose $u_{j} \geq 1$ for $j \leq \ell$ and $u_{j} \leq 1$ for $j>\ell$. Then

$$
\sum_{j>\ell}^{\infty} u_{j}^{k} e^{-\alpha u_{j}^{2} / 4} \leq \sum_{j>\ell}^{\infty} u_{j}^{k} \leq \frac{2^{k}}{2^{k}-1} .
$$

For the other terms we use

$$
u^{k+1} e^{-\alpha u^{2} / 4} \leq C
$$

for some constant $C$. Then

$$
\sum_{j \leq \ell} u_{j}^{k} e^{-\alpha u_{j}^{2} / 4} \leq C \sum_{j \leq \ell} u_{j}^{-1} \leq 2 C
$$

since $u_{j-1}^{-1} \leq \frac{1}{2} u_{j}^{-1}$ and $u_{\ell}^{-1} \leq 1$. This completes the proof.

Theorem 4.3. Let $F$ be a solution of the harmonic map heat flow from $M^{m}$ to $N^{n}$ on $0 \leq t<T$ with energy bounded by $E_{0}$. There exists a closed set $S$ with finite $m-2$ dimensional measure such that $F(x, t)$ converges smoothly to a limit $F(x, T)$ as $t \rightarrow T$ on compact sets in $M-S$. Moreover there exists a constant $C$ depending only on $M, N$ and $E_{0}$ such that if $U$ is any open set containing $S$ then

$$
\mathcal{H}_{m-2}(S) \leq C \liminf _{t \rightarrow} \int_{U}|D F|^{2} .
$$


Proof. We have already seen by Corollary 3.3 that there exists an $\varepsilon>0$ and a $\beta>0$ such that $F$ extends smoothly to a neighborhood of $X$ unless

$$
(T-t) \int_{M}|D F(x, t)|^{2} k_{(X, T)}(x, t) d x \geq \varepsilon
$$

for all $t$ in $T-\beta \leq t<T$. The sets $S$ of all such $X$ is clearly closed. If $S$ has finite $m-2$ dimensional measure, we can estimate it as follows. Pick $\tilde{S}$ by Theorem 4.2 with $\mathcal{H}_{m-2}(\tilde{S}) \geq \frac{1}{2} \mathcal{H}_{m-2}(S)$ and with

$$
k_{(\tilde{S}, T)}(x, t)=f_{\tilde{S}}(x, T-t)
$$

the backward solution to the heat equation satisfying

$$
k_{(\tilde{S}, T)}(x, t) \leq C /(T-t)
$$

for all $t$ in some interval $T-\gamma \leq t<T$ for some $\gamma>0$. In integrating the previous bound over $X \in \tilde{S}$ gives

$$
(T-t) \int_{M}|D F(x, t)|^{2} k_{(\tilde{S}, T)}(x, t) d x \geq \varepsilon \mathcal{H}_{m-2}(\tilde{S})
$$

for all $t$ in $T-\beta \leq t<T$. This gives

$$
\varepsilon \mathcal{H}_{m-2}(\tilde{S}) \leq C \int_{M}|D F(x, t)|^{2} d x \leq C E_{0}
$$

which makes

$$
H_{m-2}(S) \leq 2 C E_{0} / \varepsilon
$$

If $S$ has infinite $m-2$ dimensional measure, we get a contradiction by repeating the previous argument starting with $S^{\prime} \subseteq S$ with large but finite measure.

Finally, if $U$ is an open sset containing $S$ we have

$$
\begin{aligned}
\varepsilon \mathcal{H}_{m-2}(\tilde{S}) & \leq(T-t) \int_{U} \mid D F(x, t)^{2} k_{(\tilde{S}, T)}(x, t) d x \\
& +(T-t) \int_{M-U}|D F(x, t)|^{2} k_{(\tilde{S}, T)}(x, t) d x .
\end{aligned}
$$

We use $k_{(\tilde{S}, T)}(x, t) \leq C /(T, t, t)$ on $U$ and note that $k_{(\tilde{S}, T)}(x, t) \rightarrow 0$ uniformly on $M-U$ as $t \rightarrow T$. Thiis shows that

$$
\varepsilon \mathcal{H}_{m \rightarrow 2}(\tilde{S}) \leq C \liminf _{t \rightarrow T} \int_{U}|D F|^{2}
$$


which completes the proof.

$\S 5$. Now we examine the singularities in greater detail by blowing them up. We suppose that $F$ has a singularity at $X \in M$ at time $T$, and satisfies the estimate

$$
|D F|^{2} \leq C /(T-t)
$$

for some constant $C$. This is typical dilation-invariant growth estimate like that assumed by Huisken in [6]. The estimates in Theorem 2.2 give dilationinvariant bounds

$$
\left|D^{k} F\right|^{2} \leq C /(T-t)^{k} .
$$

We then proceed to blow up the solution $F(x, t)$ by expanding in space and time. We dilate $M$ only, but not $N$. Then a subsequence will converge to a map

$$
\bar{F}: R^{m} \times[-\infty, 0) \rightarrow N
$$

where we identify $R^{m}$ with the tangent space to $M$ at $X$ and let

$$
\bar{F}(u, v)=\lim _{\lambda \rightarrow 0} F\left(\exp _{X} \lambda u, T+\lambda^{2} v\right) .
$$

We see that $\bar{F}$ is smooth and also satisfies the harmonic map heat flow

$$
\frac{\partial \bar{F}}{\partial t}=\Delta \bar{F}
$$

and the dilation-invariant estimates

$$
\left|D^{k} \bar{F}\right| \leq C_{k} /|t|^{k}
$$

Moreover it turns out that $\bar{F}$ is itself dilation invariant.

Theorem 5.1. The map $\bar{F}$ satisfies

$$
\bar{F}\left(\lambda u, \lambda^{2} v\right)=\bar{F}(u, v)
$$

and so is dilation invariant.

Proof. From the monotonicity result in Corollary 1.4 we have

$$
\int_{T-1}^{T}(T-t) \int_{M}\left|\frac{\partial F}{\partial t}+\frac{D k}{k} \cdot D F\right|^{2} k \leq C<\infty
$$


where $k$ is the solution of the backwards heat kernel on $M$ starting from a $\delta$-function at $X$ at time $T$. Then for any $\varepsilon>0$ we can find $\delta>0$ such that

$$
\int_{T-\delta}^{T}(T-t) \int_{M}\left|\frac{\partial F}{\partial t}+\frac{D k}{k} \cdot D F\right|^{2} k \leq \varepsilon
$$

Now these estimates are dilation invariant. Moreover a subsequence of the dilations of $k$ will converge to a solution $\bar{k}$ of the backwards heat equation on $R^{m}$. Then we get

$$
\int_{-\infty}^{0}|t| \int_{R^{m}}\left|\frac{\partial \bar{F}}{\partial t}+\frac{D \bar{k}}{k} \cdot D \bar{F}\right|^{2}=0
$$

which shows that

$$
\frac{\partial \bar{F}}{\partial t}+\frac{D \bar{k}}{k} \cdot D \bar{F}=0
$$

Now in fact $D \bar{k} / \bar{k}=x / 2 t$, so we get that $\bar{F}$ is dilation invariant. Indeed at time $t=-1$ we see $\bar{F}: R^{m} \rightarrow N$ satisfies

$$
\triangle \bar{F}=\frac{1}{2} x \cdot D \bar{F}
$$

which is an elliptic equation.

We call a solution $\bar{F}$ of the harmonic map heat flow satisfying the dilation-invariant condition

$$
\bar{F}(x, t)=\bar{F}\left(\lambda x, \lambda^{2} t\right)
$$

a homothetic solition, since it moves without changing its shape except by dilation. This proves that if $F$ satisfies the dilation-invariant growth condition $|D F|^{2} \leq C /(T-t)$ then every singularity is asymptotic to a homothetic solition. Moreover if $X$ is a singular point, then from Corollary 3.3 we know that for all $t$ in $T-\beta \leq t<T$ we have

$$
(T-t) \int_{M}|D F(x, t)|^{2} k_{(X, T)}(x, t) d x \geq \varepsilon
$$

for a fixed $\varepsilon>0$, and this estimate is dilation invariant. We have assumed

$$
(T-t)|D F(x, t)|^{2} \leq C
$$

and if we take $\rho$ large enough then

$$
\int_{d(x, x) \geq \rho \sqrt{T-t}} k_{(X, T)}(x, t) d x \leq \varepsilon / 2 C
$$


since in the limit of the blow-up the heat kernels on the manifold approach that of Euclidean space. Then

$$
(T-t) \int_{d(x, x) \leq \rho \sqrt{T-t}}|D F(x, t)|^{2} k_{(X, T)}(x, t) d x \geq \varepsilon / 2
$$

and now the same will hold in the limit; so

$$
|t| \int_{|x-x| \leq \rho \sqrt{|t|}}|D \bar{F}(x, t)|^{2} \bar{k}_{(x, 0)}(x, t) d x \geq \varepsilon / 2
$$

which shows the soliton $\vec{F}$ is not constant. In part 1 we gave an example of such a soliton forming for maps of $S^{3}$ to $S^{3}$ which have a rotational symmetry. This example has a singular set of codimension 3 . Can we find an example of rapidly forming singularity where the singular set actually has codimension 2 ? The singularities which form on a surface in finite time are slowly forming, in the sense that $(T-t)|D F|^{2}$ is unbounded.

\section{References.}

[1] K.-C. Chang, W.-Y. Ding and R. Ye,Finite time blow-up of the heat flow of harmonic maps from surfaces, preprint.

[2] Y. Chen and M. Struwe(1989), Existence and partial regularity for the solutions to evolution problems for harmonic maps, Math. Z.,201,83-103.

[3] J. Eells and J.H. Sampson (1964), Harmonic mappings of Riemannian manifolds, Amer. J. Math., 86,109-160.

[4] R.S. Hamilton, Monotonicity principles for evolution equations in geometry, preprint.

[5] R.S. Hamilton, A matrix Harnack estimate for the heat equation, preprint.

[6] G. Huisken(1990),Asymptotic Behavior for singularities of the mean curvature flow, J. Diff. Geom., 31, 285-299.

[7] P. Li and S.-T. Yau(1986), On the parabolic kernel of the Schrödinger operator, Acta Math., 156, 153-201.

[8] W.-X. Shi(1989), Deforming the metric on complete Riemmanian manifolds, J. Diff. Geom., 30, 223-301. 
[9] W.-X. Shi(1989), Ricci deformation of the metric on complete non-compact Riemannian manifolds, J. Diff. Geom., 30, 303-394.

[10] M. Struwe(1985), On the evolution of harmonic maps of Riemannian surfaces, Comm. Math. Helv., 60, 558-581.

[11] M.Struwe(1988), On the evolution of harmonic maps in higher dimensions, J. Diff. Geom., 28, 485-502.

RECEIVED DECEMBER 15, 1993.

DEPARTMENT OF MATHEMATICS

UNIVERSity of CALIFornia, SAN Diego

LA Jolla, CA 92093 